\title{
Synthesis of 2-aryl substituted 2,3-dihydroquinazoline-4(1H)-ones under solvent free conditions using ionic liquid as a mild and efficient catalyst
}

\author{
Obaiah Obaiah, Nandeesh Nagalingaiah Kebbahalli, Raghavendra Manchigaiah Goravanahalli, \\ Pavankumar Siddalingaiah Chottanahalli, Rangappa Subbegowda Kanchugarakoppal, \\ and Mantelingu Kempegowda *
}

Department of Studies in Chemistry, University of Mysore, Manasagangotri, Mysore, 570006, India

*Corresponding author at: Department of Studies in Chemistry, University of Mysore, Manasagangotri, Mysore, 570006, India Tel.: +91.821.2412191. Fax: +91.821.2412191. E-mail address: kmantelingu@yahoo.com (M. Kempegowda).

\section{COMMUNICATION INFORMATION}

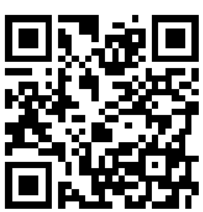

DOI: $10.5155 /$ eurjchem.5.4.671-675.1071

Received: 10 April 2014

Received in revised form: 14 May 2014

Accepted: 13 June 2014

Online: 31 December 2014

\section{KEYWORDS}

\section{Aldehyde}

Ionic liquid

Anthranilamide

Choline chloride

Solvent free condition

2,3-Dihydroquinazoline 4(1H)-ones

\section{Introduction}

2,3-Dihydroquinazolin-4(1H)-one derivatives are important heterocyclic compounds. These are widely used in biological and pharmaceutical activities [1]. The quinazolinones display wide range of biological activities such as antitumor [2,3], antidefibrillatory [4], antidepressant [5], analgesic [6], diuretic [7], antihistamine [8], vasodilating [9], antihypertensive [10], CNS stimulant [11], tranquilizing [12] and antianxietic [13]. Some representative drug molecules having quinazoline skeleton are shown in Figure 1. These quinazolinones also act as plant growth regulators [14].<smiles>O=C1NC(=O)C2C=CC=CC2N1</smiles>

BENZOURACIL

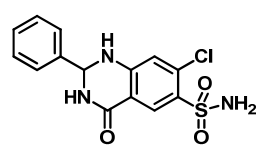

FENQUIZONE

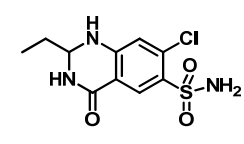

QUINETHAZONE
Figure 1. Some marketed drugs with quinazolinone skeleton.

As a consequence, in recent years these 2,3-dihydro quinazolin-4(1H)-ones have become attractive targets in synthetic chemistry. Development of novel synthetic methodologies to facilitate preparation of desired molecules has become an intense area of research. In this regard, efforts have been made constantly to introduce new methodologies that are efficient and more compatible with the environment. Consequently enormous number of synthetic methods has been developed for the construction of quinazoline alkaloids $[15,16]$. Among these, condensation of 2-aminobezamide with aldehydes is one of the simple and direct methods for the synthesis of 2,3-dihydroquinazolin-4(1H)-ones. The classical methods for the synthesis of 2,3-dihydroquinazolin-4(1H)-ones are associated with various acid catalysts such as $\mathrm{PTSA} / \mathrm{NaHSO}_{3}, \mathrm{TiCl}_{4} / \mathrm{Zn}$, $\mathrm{CuCl}_{2}$, ionic liquid, TFA, ammonium chloride and chiral phosphoric acid $[17,18]$. Although these methods are very useful for the synthesis of 2,3-dihydroquinazolin-4(1H)-ones but the reactions involved have major limitations such as expensive reagents, lengthy reaction times, high temperature requirement and also tedious work-up procedures. Therefore, the development of novel method to overcome the limitations for the synthesis of quinazolin-4(1H)-ones is of great importance because of their biological and pharmaceutical importance. 


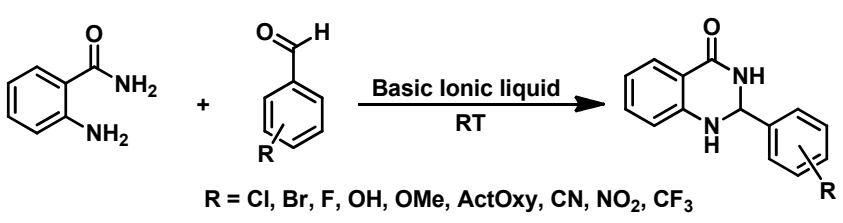

Scheme 1

Over the past decade utility of ionic liquids in catalysis and as neoteric solvent for various synthetic processes has been well recognized by the chemists all over the world. Due to their chemical properties, such as recyclability, negligible vapor pressure, ability to dissolve wide range of substrates and catalysts, thermal stability. Ionic liquids have been considered as viable alternatives to the conventional volatile organic solvents [19-23]. Protic and Bronsted basic liquids, in particular, have received increasing attention for carrying out organic transformations as they can replace volatile organic solvents as well as highly acidic catalytic systems [24-25]. Hence uses of ionic liquids have been found advantageous as they usually involve in simple reaction processes as compared to most of the traditional methods [23].

\section{Experimental}

\subsection{Materials and methods}

Works relating to analytical thin layer chromatography were performed using E. Merck silica gel $60 \mathrm{~F}_{254}$ aluminum plates and were visualized with UV light. The following mobile phases employed for TLC were chloroform, methanol and hexane, and ethyl acetate in different ratios. The instrumental techniques adopted for the characterization of the newly synthesized compounds include ${ }^{1} \mathrm{H}$ and ${ }^{13} \mathrm{C}$ NMR and mass spectroscopy. ${ }^{1} \mathrm{H}$ and ${ }^{13} \mathrm{C}$ NMR spectra were recorded on a Bruker WM (400 and $300 \mathrm{MHz}$ ) spectrometer in $\mathrm{CDCl}_{3}$ or DMSO- $d_{6}$ solution using tetramethylsilane (TMS) as internal standard. Chemical shifts were recorded in ppm relative to TMS. Mass and purity were recorded on LC-MSD-Trap-XCT (Agilent technologies Inc).

\subsection{Synthesis}

Mixture of choline chloride ( $2 \mathrm{~g}, 0.0143 \mathrm{mmol})$ and urea (1.72 g, $0.0286 \mathrm{mmol}$ ) was heated for $100-200{ }^{\circ} \mathrm{C}$, and at certain temperature it formed into liquid. To this reaction mixture 2-amino benzamide $(0.2 \mathrm{~g}, 0.00147 \mathrm{mmol})$ and 4formylphenyl acetate $(0.241 \mathrm{~g}, 0.00147 \mathrm{mmol})$ were added (Scheme 1). The mixture was stirred for $3 \mathrm{~h}$. Reaction completion was confirmed by TLC. It was extracted with ethyl acetate. The combined organic layer was washed with water, and saturated brine solution followed by using over anhydrous $\mathrm{Na}_{2} \mathrm{SO}_{4}$. The combined organic layer was evaporated under vacuum pressure and the resulting crude product was purified by column chromatography with ethyl acetate in hexane as eluent which afforded the desired product. The products were identified by ${ }^{1} \mathrm{H}$ NMR, ${ }^{13} \mathrm{C}$ NMR and LC/MS, showed good agreement with the assigned structures.

4-(4-Oxo-1,2,3,4-tetrahydroquinazolin-2-yl)phenyl acetate (Table 1, Entry 1): Color: White solid. Yield: $80 \% .{ }^{1} \mathrm{H}$ NMR $(300$ MHz, DMSO- $\left.d_{6}, \delta, \mathrm{ppm}\right): 8.27(\mathrm{~s}, 1 \mathrm{H}, \mathrm{NH}), 7.58-7.61(\mathrm{~d}, 1 \mathrm{H}, J=$ 7.6 Hz, Ar-H),7.49-7.52 (d, $2 \mathrm{H}, J=8.4 \mathrm{~Hz}, \mathrm{Ar}-\mathrm{H}), 7.20-7.25$ (t, $2 \mathrm{H}, J=7.3 \mathrm{~Hz}, \mathrm{Ar}-\mathrm{H}), 7.11-7.14(\mathrm{~d}, 2 \mathrm{H}, J=8.4 \mathrm{~Hz}, \mathrm{Ar}-\mathrm{H}), 6.71-$ $6.74(\mathrm{~d}, 1 \mathrm{H}, J=8.1 \mathrm{~Hz}, \mathrm{Ar}-\mathrm{H}), 6.64-6.69(\mathrm{t}, 1 \mathrm{H}, J=7.5 \mathrm{~Hz}, \mathrm{CH}-\mathrm{NH}-$ ), 5.76 (s, $1 \mathrm{H}, \mathrm{NH}), 2.27$ (s, 3H, $\mathrm{CH}_{3}-\mathrm{CO}-\mathrm{O}-$ ). ${ }^{13} \mathrm{C}$ NMR $(75 \mathrm{MHz}$, DMSO- $\left.d_{6}, \delta, \mathrm{ppm}\right): 169.61,164.00,150.99,148.25,239.53$, $133.80,129.66,128.51,127.82,126.32,122.18,117.65,115.36$, 114.80, 66.58, 21.28. MS (m/z): $283.20\left[\mathrm{M}^{+}+\mathrm{H}\right]$. Anal. calcd. for
$\mathrm{C}_{16} \mathrm{H}_{14} \mathrm{~N}_{2} \mathrm{O}_{3}: \mathrm{C}, 68.07 ; \mathrm{H}, 5.00 ; \mathrm{N}, 9.92$. Found: $\mathrm{C}, 68.02 ; \mathrm{H}, 4.98$; $\mathrm{N}, 9.87 \%$.

2-(2, 4-Dimethylphenyl)-2, 3-dihydroquinazolin-4(1H)-one (Table 1, Entry 2): Color: White solid. Yield: $80 \% .{ }^{1} \mathrm{H}$ NMR $(300$ MHz, DMSO- $\left.d_{6}, \delta, \mathrm{ppm}\right): 7.99$ (s, 1H, NH), 7.61-7.64 (d, $1 \mathrm{H}, J=$ $10.28 \mathrm{~Hz}, \mathrm{Ar}-\mathrm{H}), 7.40-7.43$ (d, $1 \mathrm{H}, J=10.64 \mathrm{~Hz}, \mathrm{Ar}-\mathrm{H}), 7.20-7.23$ (t, $1 \mathrm{H}, J=10.14 \mathrm{~Hz}, \mathrm{Ar}-\mathrm{H}), 7.01-7.04(\mathrm{~s}, 2 \mathrm{H}, \mathrm{Ar}-\mathrm{H}), 6.65-6.79(\mathrm{~m}$, $3 \mathrm{H}, \mathrm{CH}-\mathrm{NH}+\mathrm{Ar}-\mathrm{H}), 5.93(\mathrm{~s}, 1 \mathrm{H}, \mathrm{NH}), 2.37\left(\mathrm{~s}, 3 \mathrm{H}, \mathrm{Ar}^{-\mathrm{CH}_{3}}\right), 2.26$ (s, 3H, Ar- $\mathrm{CH}_{3}$ ). ${ }^{13} \mathrm{C}$ NMR (75 MHz, DMSO-d6, $\delta, \mathrm{ppm}$ ): 164.52, $149.01,138.10,136.35,135.57,133.60,131.71,129.66,128.25$, 127.87, 126.86, 117.59, 114.91, 64.51, 21.01, 19.16. MS (m/z): $253.30\left[\mathrm{M}^{++} \mathrm{H}\right]$. Anal. calcd. for $\mathrm{C}_{16} \mathrm{H}_{16} \mathrm{~N}_{2} \mathrm{O}: \mathrm{C}, 76.16 ; \mathrm{H}, 6.39 ; \mathrm{N}$, 11.10. Found: C, 76.12; H, 6.38; N, $11.12 \%$.

2-(4-Methoxyphenyl)-2, 3-dihydroquinazolin-4(1H)-one (Table 1, Entry 3): Color: White solid. Yield: $81 \% .{ }^{1} \mathrm{H}$ NMR (300 MHz, DMSO- $\left.d_{6}, \delta, \mathrm{ppm}\right): 8.16(\mathrm{~s}, 1 \mathrm{H}, \mathrm{NH}), 7.57-7.60(\mathrm{~d}, 1 \mathrm{H}, J=$ 7.72 Hz, Ar-H), 7.38-7.41 (d, 2H, J = 8.67 Hz, Ar-H), 7.19-7.24 (t, $1 \mathrm{H}, J=7.83 \mathrm{~Hz}, \mathrm{Ar}-\mathrm{H}), 6.98(\mathrm{~s}, 1 \mathrm{H}, \mathrm{Ar}-\mathrm{H}), 6.91-6.94(\mathrm{~d}, 2 \mathrm{H}, J=$ $8.7 \mathrm{~Hz}, \mathrm{Ar}-\mathrm{H}), 6.70-6.73(\mathrm{~d}, 1 \mathrm{H}, J=7.95 \mathrm{~Hz}, \mathrm{Ar}-\mathrm{H}), 6.63-6.68(\mathrm{t}$, $1 \mathrm{H}, J=7.23 \mathrm{~Hz}, \mathrm{CH}-\mathrm{NH}), 5.68(\mathrm{~s}, 1 \mathrm{H}, \mathrm{NH}), 3.73\left(\mathrm{~s}, 3 \mathrm{H}, \mathrm{CH}_{3}-\mathrm{O}-\right)$. ${ }^{13} \mathrm{C}$ NMR $\left(75 \mathrm{MHz}, \mathrm{DMSO}-d_{6}, \delta, \mathrm{ppm}\right): 164.13,159.89,148.46$, 133.94, 128.65, 127.79, 117.52, 115.46, 114.86, 114.09, 66.75, 55.63. MS (m/z): $255.28\left[\mathrm{M}^{+}+\mathrm{H}\right]$. Anal. calcd. for $\mathrm{C}_{15} \mathrm{H}_{14} \mathrm{~N}_{2} \mathrm{O}_{2}$ : C, 70.85; H, 5.55; N, 11.02. Found: C, 70.80; H, 5.53; N, $11.00 \%$.

2-(3, 4-Dimethoxyphenyl)-2, 3-dihydroquinazolin-4(1H)-one (Table 1, Entry 4): Color: White solid. Yield: $82 \% .{ }^{1} \mathrm{H}$ NMR $(300$ MHz, DMSO- $\left.d_{6}, \delta, \mathrm{ppm}\right): 8.06(\mathrm{~s}, 1 \mathrm{H} \mathrm{NH}), 7.60-7.62(\mathrm{~d}, 1 \mathrm{H}, J=$ 7.71 Hz, Ar-H), 7.15-7.26 (m, 3H, Ar-H), 6.83 (s, 1H, Ar-H), 6.65$6.73(\mathrm{~m}, 3 \mathrm{H}, \mathrm{CH}-\mathrm{NH}, \mathrm{Ar}-\mathrm{H}), 5.99$ (s, 1H, NH), 3.80 (s, 3H Ph$\left.\mathrm{OCH}_{3}\right), 3.70\left(\mathrm{~s}, 3 \mathrm{H}, \mathrm{Ph}-\mathrm{OCH}_{3}\right) .{ }^{13} \mathrm{C}$ NMR $\left(75 \mathrm{MHz}, \mathrm{DMSO}-d_{6}, \delta\right.$, ppm): 164.75, 153.20, 146.69, 145.49, 134.54, 134.18, 128.60, $121.62,119.53,117.02,116.72,115.08,114.70,61.44,56.17$. MS (m/z): 285.11 [M++ H]. Anal. calcd. for $\mathrm{C}_{16} \mathrm{H}_{16} \mathrm{~N}_{2} \mathrm{O}_{3}$ : C, 67.59; H, 5.67; N, 9.85. Found: C, 67.56; H, 5.68; N, 9.82\%.

2-(4-Fluorophenyl)-2, 3-dihydroquinazolin-4(1H)-one (Table 1, Entry 5): Color: White solid. Yield: $88 \% .{ }^{1} \mathrm{H}$ NMR $(300 \mathrm{MHz}$, DMSO- $\left.d_{6}, \delta, \mathrm{ppm}\right): 8.26(\mathrm{~s}, 1 \mathrm{H}, \mathrm{NH}), 7.58-7.60(\mathrm{~d}, 1 \mathrm{H}, J=7.47$ $\mathrm{Hz}, \mathrm{Ar}-\mathrm{H}), 7.49-7.54(\mathrm{~m}, 2 \mathrm{H}, \mathrm{Ar}-\mathrm{H}), 7.17-7.25$ (m, 3H, Ar-H), 7.08 (s, 1H, Ar-H), 6.71-6.74 (d, 1H, J = 8.04 Hz, Ar-H), 6.64-6.69 $(\mathrm{t}, 1 \mathrm{H}, J=7.41 \mathrm{~Hz}, \mathrm{CH}-\mathrm{NH}), 5.75(\mathrm{~s}, 1 \mathrm{H}, \mathrm{NH}) .{ }^{13} \mathrm{C}$ NMR $(75 \mathrm{MHz}$, DMSO- $\left.d_{6}, \delta, \mathrm{ppm}\right): 163.79,161.36,148.27,138.25,133.83$, $129.50,127.83,117.72,115.43,115.41,114.91,66.39$. MS (m/z): $243.24\left[\mathrm{M}^{+}+\mathrm{H}\right]$. Anal. calcd. for $\mathrm{C}_{14} \mathrm{H}_{11} \mathrm{FN}_{2} \mathrm{O}: \mathrm{C}, 69.41 ; \mathrm{H}$, 4.58; N, 11.56. Found: C, 69.40; H, 4.58; N, $11.53 \%$.

2-(4-Chlorophenyl)-2, 3-dihydroquinazolin-4(1H)-one (Table 1, Entry 6): Color: White solid. Yield: $87 \% .{ }^{1} \mathrm{H}$ NMR $(300 \mathrm{MHz}$, DMSO- $\left.d_{6}, \delta, \mathrm{ppm}\right): 8.32(\mathrm{~s}, 1 \mathrm{H}, \mathrm{NH}), 7.58-7.60(\mathrm{~d}, 2 \mathrm{H}, J=7.68$ $\mathrm{Hz}, \mathrm{Ar}-\mathrm{H}), 7.42-7.51(\mathrm{~m}, 3 \mathrm{H}, \mathrm{Ar}-\mathrm{H}), 7.20-7.25(\mathrm{t}, 1 \mathrm{H}, J=7.23 \mathrm{~Hz}$, Ar-H), 7.12 (s, $1 \mathrm{H}, \mathrm{Ar}-\mathrm{H}), 6.71-6.74(\mathrm{~d}, 1 \mathrm{H}, J=8.10 \mathrm{~Hz}, \mathrm{Ar}-\mathrm{H})$, 6.64-6.69 (t, 1H, $J=7.53 \mathrm{~Hz}, \mathrm{CH}-\mathrm{NH}), 5.75(\mathrm{~s}, 1 \mathrm{H}, \mathrm{NH}) .{ }^{13 \mathrm{C}} \mathrm{NMR}$ (75 MHz, DMSO- $\left.d_{6}, \delta, \mathrm{ppm}\right): 163.79,161.36,148.27,138.27$, $138.24,133.83,129.55,129.46,127.83,117.72,115.45,111.41$, 114.91, 66.39. MS $(\mathrm{m} / \mathrm{z}): 259.70\left[\mathrm{M}^{+}+\mathrm{H}\right]$. Anal. calcd. for $\mathrm{C}_{14} \mathrm{H}_{11} \mathrm{ClN}_{2} \mathrm{O}: \mathrm{C}, 65.00 ; \mathrm{H}, 4.29 ; \mathrm{N}, 10.83$. Found: C, 65.02; H, 4.26; $\mathrm{N}, 10.82 \%$.

4-(4-Oxo-1, 2, 3, 4-tetrahydroquinazolin-2-yl)benzonitrile (Table 1, Entry 7): Color: White solid. Yield: $90 \% .{ }^{1} \mathrm{H}$ NMR $(300$ MHz, DMSO- $\left.d_{6}, \delta, p p m\right): 8.47(\mathrm{~s}, 1 \mathrm{H}, \mathrm{NH}), 7.85-7.87(\mathrm{~d}, 2 \mathrm{H}, J=$ $8.4 \mathrm{~Hz}, \mathrm{Ar}-\mathrm{H}), 7.64-7.66(\mathrm{~d}, 2 \mathrm{H}, J=8.4 \mathrm{~Hz}, \mathrm{Ar}-\mathrm{H}), 7.58-7.60(\mathrm{t}$, $1 \mathrm{H}, J=7.6 \mathrm{~Hz}, \mathrm{Ar}-\mathrm{H}), 7.23-7.28$ (m, 2H, Ar-H), 6.74-6.76 (d, 1H, 
Obaiah et al. / European Journal of Chemistry 5 (4) (2014) 671-675

673

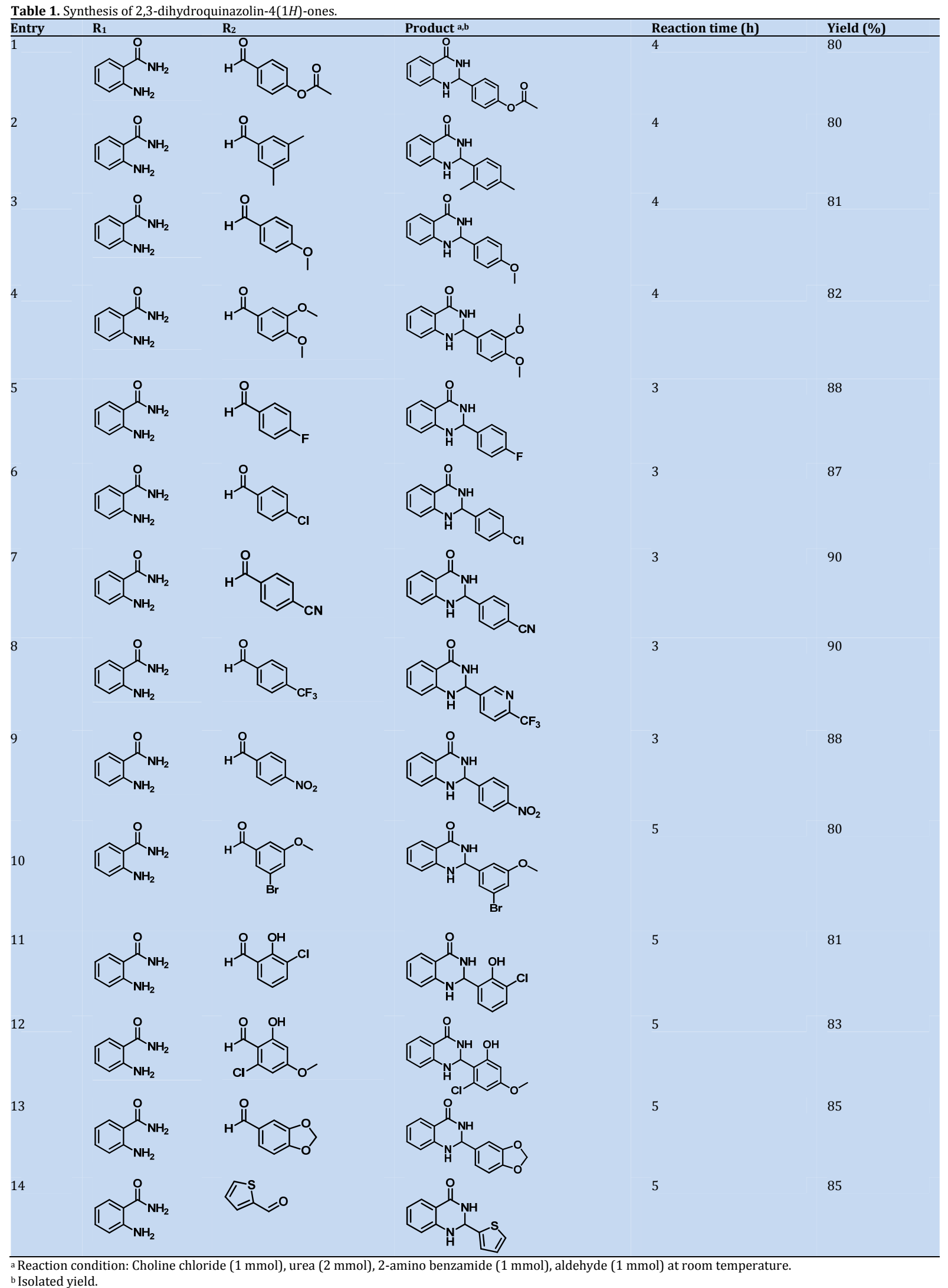




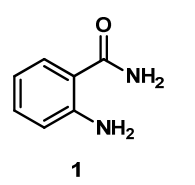

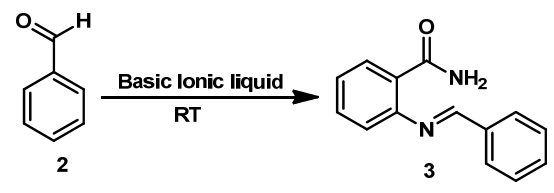

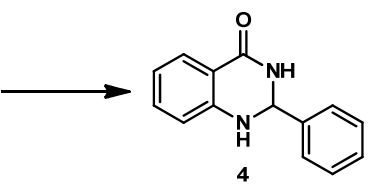

Scheme 2

$J=8.0 \mathrm{~Hz}, \mathrm{Ar}-\mathrm{H}), 6.66-6.69(\mathrm{t}, 1 \mathrm{H}, J=7.2 \mathrm{~Hz}, \mathrm{CH}-\mathrm{NH}), 5.84(\mathrm{~s}$, $1 \mathrm{H}, \mathrm{NH}) .{ }^{13} \mathrm{C}$ NMR $\left(75 \mathrm{MHz}\right.$, DMSO- $\left.d_{6}, \delta, \mathrm{ppm}\right): 163.30,147.34$, 147.31, 133.53, 132.39, 127.67, 127.37, 118.64, 117.40, 114.88, 114.49, 111.04, 65.50. MS $(\mathrm{m} / \mathrm{z}): 250.26\left[\mathrm{M}^{+}+\mathrm{H}\right]$. Anal. calcd. for $\mathrm{C}_{15} \mathrm{H}_{11} \mathrm{~N}_{3} \mathrm{O}$ : C, 72.28; $\mathrm{H}, 4.45 ; \mathrm{N}, 16.86$; Found: $\mathrm{C}, 72.27 ; \mathrm{H}$, $4.40 ; \mathrm{N}, 16.84 \%$.

2-(6-(Trifluoromethyl)pyridin-3-yl)-2, 3-dihydroquinazolin4(1H)-one (Table 1, Entry 8): Color: White solid. Yield: $90 \% .{ }^{1} \mathrm{H}$ NMR (300 MHz, DMSO- $\left.d_{6}, \delta, \mathrm{ppm}\right): 8.86(\mathrm{~s}, 1 \mathrm{H}, \mathrm{Ar}-\mathrm{H}), 8.50$ (s, $1 \mathrm{H}, \mathrm{NH}), 8.14-8.16(\mathrm{~d}, 1 \mathrm{H}, J=7.2 \mathrm{~Hz}, \mathrm{Ar}-\mathrm{H}), 7.95-7.97$ (d, $1 \mathrm{H}, J=$ $8.0 \mathrm{~Hz}, \mathrm{Ar}-\mathrm{H}$ ), 7.61-7.63 (d, 1H, J = 7.2 Hz, Ar-H), 7.26-7.30 (m, $2 \mathrm{H}, \mathrm{Ar}-\mathrm{H}), 6.76-6.78(\mathrm{~d}, 1 \mathrm{H}, J=8.0 \mathrm{~Hz}, \mathrm{Ar}-\mathrm{H}), 6.70-6.74(\mathrm{t}, 1 \mathrm{H}, J$ $=7.2 \mathrm{~Hz}, \mathrm{CH}-\mathrm{NH}-), 5.98(\mathrm{~s}, 1 \mathrm{H}, \mathrm{NH}) .{ }^{13} \mathrm{C}$ NMR $\left(75 \mathrm{MHz}\right.$, DMSO- $d_{6}$, $\delta, \mathrm{ppm}): 163.38,149.02,147.33,146.17,140.84,136.76$, $133.63,127.44,122.92,120.20,117.77,115.00,114.64,63.99$. MS $(m / z): 294.24\left[\mathrm{M}^{+}+\mathrm{H}\right]$. Anal. calcd. for $\mathrm{C}_{14} \mathrm{H}_{10} \mathrm{~F}_{3} \mathrm{~N}_{3} \mathrm{O}$ : C, 57.34; H, 3.44; N, 14.33. Found: C, 57.34; H, 3.42; N, 14.32\%.

2-(4-Nitrophenyl)-2, 3-dihydroquinazolin-4(1H)-one (Table 1, Entry 9): Color: Yellow solid. Yield: $88 \% .{ }^{1} \mathrm{H}$ NMR (300 MHz, DMSO- $\left.d_{6}, \delta, \mathrm{ppm}\right): 8.50(\mathrm{~s}, 1 \mathrm{H}, \mathrm{NH}), 8.22-8.35(\mathrm{~d}, 2 \mathrm{H}, J=8.61$ $\mathrm{Hz}, \mathrm{Ar}-\mathrm{H}), 7.71-7.74$ (d, $2 \mathrm{H}, J=8.5 \mathrm{~Hz}, \mathrm{Ar}-\mathrm{H}), 7.58-7.60$ (d, 1H, $J$ $=7.62 \mathrm{~Hz}, \mathrm{Ar}-\mathrm{H}), 7.22-7.27(\mathrm{t}, 2 \mathrm{H}, J=7.95 \mathrm{~Hz}, \mathrm{Ar}-\mathrm{H}), 6.73-6.76$ (d, $1 \mathrm{H}, J=8.07 \mathrm{~Hz}, \mathrm{Ar}-\mathrm{H}), 6.64-6.69(\mathrm{t}, 1 \mathrm{H}, J=7.47 \mathrm{~Hz}, \mathrm{CH}-\mathrm{NH}-$ ), $5.87(\mathrm{~s}, 1 \mathrm{H}, \mathrm{NH}) .{ }^{13} \mathrm{C}$ NMR (75 MHz, DMSO- $\left.d_{6}, \delta, \mathrm{ppm}\right): 163.72$, $149.78,147.89,147.89,147.68,143.00,128.48,127.86,124.02$, $117.91,115.37,115.00,67.75$. MS $(\mathrm{m} / z): 270.08\left[\mathrm{M}^{++} \mathrm{H}\right]$. Anal. calcd. for $\mathrm{C}_{14} \mathrm{H}_{11} \mathrm{~N}_{3} \mathrm{O}_{3}, \mathrm{C}, 62.45 ; \mathrm{H}, 4.12 ; \mathrm{N}, 15.61$. Found: C, 62.43; H, 4.10; N, $15.60 \%$.

2-(3-Bromo-5-methoxyphenyl)-2, 3-dihydroquinazolin4(1H)-one (Table 1, Entry 10): Color: White solid. Yield: $80 \%$. ${ }^{1} \mathrm{H}$ NMR $\left(300 \mathrm{MHz}\right.$, DMSO- $\left.d_{6}, \delta, \mathrm{ppm}\right): 8.11(\mathrm{~s}, 1 \mathrm{H}, \mathrm{NH}), 7.62-$ $7.64(\mathrm{~d}, 1 \mathrm{H}, J=7.56 \mathrm{~Hz}, \mathrm{Ar}-\mathrm{H}), 7.49-7.52(\mathrm{~d}, 1 \mathrm{H}, J=8.72 \mathrm{~Hz}, \mathrm{Ar}-$ H), $7.45(\mathrm{~s}, 1 \mathrm{H}, \mathrm{Ar}-\mathrm{H}), 7.23-7.27$ (t, $1 \mathrm{H}, J=7.04 \mathrm{~Hz}, \mathrm{Ar}-\mathrm{H}), 7.03-$ $7.05(\mathrm{~d}, 1 \mathrm{H}, J=8.72 \mathrm{~Hz}, \mathrm{Ar}-\mathrm{H}), 6.88(\mathrm{~s}, 1 \mathrm{H}, \mathrm{Ar}-\mathrm{H}), 6.76-6.78(\mathrm{~d}$, $1 \mathrm{H}, J=8.08 \mathrm{~Hz}, \mathrm{Ar}-\mathrm{H}), 6.67-6.71(\mathrm{t}, 1 \mathrm{H}, J=7.36 \mathrm{~Hz}, \mathrm{CH}-\mathrm{NH}-)^{-}$, $5.98(\mathrm{~s}, 1 \mathrm{H}, \mathrm{NH}), 3.83\left(\mathrm{~s}, 3 \mathrm{H}, \mathrm{Ar}-\mathrm{OCH}_{3}\right) .{ }^{13} \mathrm{C}$ NMR $(75 \mathrm{MHz}$, DMSO- $\left.d_{6}, \delta, \mathrm{ppm}\right): 164.07,156.24,148.11,133.86,132.58$, 131.84, 129.91, 131.84, 129.91, 127.78, 117.76, 115.01, 114.97, 114.13, 112.08, 61.19, 56.41. MS (m/z): $334.17\left[\mathrm{M}^{+}+\mathrm{H}\right]$. Anal. calcd. for $\mathrm{C}_{15} \mathrm{H}_{13} \mathrm{BrN}_{2} \mathrm{O}_{2}$ : C, 54.07; $\mathrm{H}, 3.93 ; \mathrm{N}, 8.41$. Found: $\mathrm{C}$, 54.06; H, 3.92; N, 8.40\%.

2-(3-Chloro-2-hydroxyphenyl)-2, 3-dihydroquinazolin-4(1H)one (Table 1, Entry 11): Color: White solid. Yield: $81 \% .{ }^{1} \mathrm{H}$ NMR (300 MHz, DMSO- $\left.d_{6}, \delta, \mathrm{ppm}\right): 8.02(\mathrm{~s}, 1 \mathrm{H}, \mathrm{NH}), 7.59-7.62(\mathrm{~d}, 1 \mathrm{H}$, $J=7.74 \mathrm{~Hz}, \mathrm{Ar}-\mathrm{H}), 7.29-7.33(\mathrm{~m}, 2 \mathrm{H}, \mathrm{Ar}-\mathrm{H}), 7.19-7.24(\mathrm{t}, 1 \mathrm{H}, J=$ $7.63 \mathrm{~Hz}, \mathrm{Ar}-\mathrm{H}$ ), 6.81-6.86 (t, $1 \mathrm{H}, J=7.8 \mathrm{~Hz}, \mathrm{Ar}-\mathrm{H}), 6.73-6.76(\mathrm{~d}$, $2 \mathrm{H}, J=8.04 \mathrm{~Hz}, \mathrm{Ar}-\mathrm{H}), 6.64-6.69(\mathrm{t}, 1 \mathrm{H}, J=7.17 \mathrm{~Hz}, \mathrm{CH}-\mathrm{NH}-)$, $6.06(\mathrm{~s}, 1 \mathrm{H}, \mathrm{NH}) .{ }^{13} \mathrm{C}$ NMR $\left(75 \mathrm{MHz}, \mathrm{DMSO}-d_{6}, \delta, \mathrm{ppm}\right): 164.28$, $150.87,148.32,133.74,130.88,129.92,127.79,126.62,121.63$, 120.50, 117.76, 115.09, 62.22. MS $(\mathrm{m} / \mathrm{z}): 275.05\left[\mathrm{M}^{+}+\mathrm{H}\right]$. Anal. calcd. for $\mathrm{C}_{14} \mathrm{H}_{11} \mathrm{ClN}_{2} \mathrm{O}_{2}: \mathrm{C}, 61.21 ; \mathrm{H}, 4.04 ; \mathrm{N}, 10.20$. Found: $\mathrm{C}$, $61.20 ; \mathrm{H}, 4.02 ; \mathrm{N}, 10.20 \%$.

2-(2-Chloro-6-hydroxy-4-methoxyphenyl)-2, 3-dihydroquina zolin-4(1H)-one (Table 1, Entry 12): Color: White solid. Yield: $83 \% .{ }^{1} \mathrm{H}$ NMR (300 MHz, DMSO-d $\left., \delta, \mathrm{ppm}\right): 9.40(\mathrm{~s}, 1 \mathrm{H}, \mathrm{Ph}-\mathrm{OH})$, $8.01(\mathrm{~s}, 1 \mathrm{H}, \mathrm{NH}), 7.61-7.63(\mathrm{~d}, 1 \mathrm{H}, J=7.6 \mathrm{~Hz}, \mathrm{Ar}-\mathrm{H}), 7.22-7.25(\mathrm{t}$, $1 \mathrm{H}, J=7.2 \mathrm{~Hz}, \mathrm{Ar}-\mathrm{H}), 6.91(\mathrm{~s}, 1 \mathrm{H}, \mathrm{Ar}-\mathrm{H}), 6.80(\mathrm{~s}, 1 \mathrm{H}, \mathrm{Ar}-\mathrm{H}), 6.75-$ $6.77(\mathrm{~d}, 2 \mathrm{H}, J=8.08 \mathrm{~Hz}, \mathrm{Ar}-\mathrm{H}), 6.66-6.70(\mathrm{t}, 1 \mathrm{H}, J=7.48 \mathrm{~Hz}, \mathrm{CH}-$ $\mathrm{NH}-), 6.01(\mathrm{~s}, 1 \mathrm{H}, \mathrm{NH}), 3.83\left(\mathrm{~s}, 3 \mathrm{H}, \mathrm{Ph}-\mathrm{OCH}_{3}\right) .{ }^{13} \mathrm{C} \mathrm{NMR}(75 \mathrm{MHz}$, DMSO- $\left.d_{6}, \delta, \mathrm{ppm}\right): 164.17,148.92,148.25,143.17,133.78$,
129.44, 127.78, 122.68, 118.81, 117.70, 115.70, 115.01, 112.38, 61.33, 56.83. MS $(\mathrm{m} / \mathrm{z})$ : $305.72\left[\mathrm{M}^{+}+\mathrm{H}\right]$. Anal. calcd. for $\mathrm{C}_{15} \mathrm{H}_{13} \mathrm{ClN}_{2} \mathrm{O}_{3}: \mathrm{C}, 59.12 ; \mathrm{H}, 4.30 ; \mathrm{N}, 9.19$. Found: C, 59.10; H, 4.29; N, 9.17\%.

2-(Benzo[d][1,3]dioxol-5-yl)-2, 3-dihydroquinazolin-4(1H)one (Table 1, Entry 13): Color: White solid. Yield: $85 \% .{ }^{1} \mathrm{H}$ NMR (300 MHz, DMSO- $\left.d_{6}, \delta, \mathrm{ppm}\right): 8.20(\mathrm{~s}, 1 \mathrm{H}, \mathrm{NH}), 7.57-7.59(\mathrm{~d}, 1 \mathrm{H}$, $J=7.71 \mathrm{~Hz}, \mathrm{Ar}-\mathrm{H}), 7.19-7.25(\mathrm{t}, 1 \mathrm{H}, J=7.5 \mathrm{~Hz}, \mathrm{Ar}-\mathrm{H}), 6.87-7.02$ (m, 4H, Ar-H), 6.71-6.73 (d, $1 \mathrm{H}, J=8.1 \mathrm{~Hz}, \mathrm{Ar}-\mathrm{H}), 6.65-6.68(\mathrm{t}$, $1 \mathrm{H}, J=7.23 \mathrm{~Hz}, \mathrm{CH}-\mathrm{NH}-), 5.99$ (s, 2H, Ph-O-CH 2$), 5.65$ (s, 1H, $\mathrm{NH}) .{ }^{13} \mathrm{C}$ NMR (75 MHz, DMSO- $\left.d_{6}, \delta, \mathrm{ppm}\right): 164.02,148.27$, 147.75, 136.03, 133.73, 127.78, 120.87, 117.59, 115.42 114.87, 108.30, 107.61, 101.56, 66.73. MS (m/z): $269.26\left[\mathrm{M}^{+}+\mathrm{H}\right]$. Anal. calcd. for $\mathrm{C}_{15} \mathrm{H}_{12} \mathrm{~N}_{2} \mathrm{O}_{3}$ : C, 67.16; $\mathrm{H}, 4.51 ; \mathrm{N}, 10.44$. Found: $\mathrm{C}$, $67.14 ; \mathrm{H}, 4.51 ; \mathrm{N}, 10.42 \%$.

2-(Thiophen-2-yl)-2, 3-dihydroquinazolin-4(1H)-one (Table 1, Entry 14): Color: White solid. Yield: $85 \% .{ }^{1} \mathrm{H}$ NMR $(300 \mathrm{MHz}$, DMSO- $\left.d_{6}, \delta, \mathrm{ppm}\right): 8.43(\mathrm{~s}, 1 \mathrm{H}, \mathrm{NH}), 7.59-7.61(\mathrm{~d}, 1 \mathrm{H}, J=7.62$ $\mathrm{Hz}, \mathrm{Ar}-\mathrm{H}$ ), 7.42-744 (d, $1 \mathrm{H}, J=4.98 \mathrm{~Hz}, \mathrm{Ar}-\mathrm{H}), 7.22-7.27$ (t, $2 \mathrm{H}, J$ $=6.9 \mathrm{~Hz}, \mathrm{Ar}-\mathrm{H}), 7.10-7.11(\mathrm{~d}, 1 \mathrm{H}, J=3.18 \mathrm{~Hz}, \mathrm{Ar}-\mathrm{H}), 6.94-6.97(\mathrm{t}$, $1 \mathrm{H}, J=84.27 \mathrm{~Hz}, \mathrm{Ar}-\mathrm{H}), 6.73-6.76(\mathrm{~d}, 1 \mathrm{H}, J=8.13 \mathrm{~Hz}, \mathrm{Ar}-\mathrm{H})$, $6.66-6.71(\mathrm{t}, 1 \mathrm{H}, J=7.38 \mathrm{~Hz}, \mathrm{CH}-\mathrm{NH}-) 6.00(\mathrm{~s}, 1 \mathrm{H}, \mathrm{NH}) .{ }^{13} \mathrm{C} \mathrm{NMR}$ (75 MHz, DMSO- $\left.d_{6}, \delta, \mathrm{ppm}\right): 147.68,146.90,133.81,127.76$, $126.90,126.32,126.13,117.96,115.36,63.01$. MS $(\mathrm{m} / \mathrm{z})$ : 231.20. $\left[\mathrm{M}^{+}+\mathrm{H}\right]$. Anal. calcd. for $\mathrm{C}_{12} \mathrm{H}_{10} \mathrm{~N}_{2} \mathrm{OS}$ : C, 62.59; H, 4.38; N, 12.16. Found: C, $62.57 ; \mathrm{H}, 4.38 ; \mathrm{N}, 12.17 \%$.

\section{Results and discussion}

In continuation numbers of our efforts in the development of novel, environ-friendly and synthetic methodologies [26-33], we report here a mild and efficient one-pot protocol for the synthesis of 2,3-dihydroquinazolin-4(1H)-one derivatives by two component reactions catalysed by ionic liquid under mild condition (Scheme 1). The reaction when tried with different aldehydes, diverse functional groups played significant roles in achieving the product yield. Aldehydes with electron withdrawing group react with 2 -amino benzaldehyde giving better yield (Table 1 entries 5-9). Whereas, aldehydes with electron releasing and both electron releasing and withdrawing give moderate yields (Table 1 entries 1-4 and 10-14). A mixture of 2 -amino benzamide $(1 \mathrm{mmol})$ and aromatic aldehyde (1 $\mathrm{mmol}$ ) in ionic liquid was stirred at room temperature for 3-4 h without an inert atmosphere. When the reaction was complete the product was purified by column chromatography. The scope of the reaction was examined with various aromatic aldehydes and the results are summarized in Table 1.

A possible mechanism of the basic ionic liquid catalysed reaction of 2-amino benzamide and aldehydes is proposed in Scheme 2. 2-Amino benzamide 1 condensation of the aromatic aldehyde $\mathbf{2}$ with ionic liquid gives imine $\mathbf{3}$ which undergoes cyclisation to afford dihydroquinazoline product 4.

\section{Conclusion}

In summary, we have developed a direct and efficient method for the preparation of 2,3-dihydroquinazolin-4(1H)ones using basic ionic liquid via cyclocondensation of 2-amino benzamide with an aldehyde. The simplicity of the procedure, easy work up, excellent yields, short reaction times, easy 
handling and use of ecofriendly, non-volatile, less expensive reagents are the advantages claimed by this method.

\section{Acknowledgements}

The authors are grateful to University Grants Commission, Government of India for financial support to Kempegowda Mantelingu for the project vide No. F. No. 39-710-2011 (SR/dated 12-01-2010).

\section{References}

[1]. Daniel, A.; Erlanson, R. S.; McDowell, T. J. Med. Chem. 2004, 47, 34633482.

[2]. Hour, M. J.; Huang, L. J.; Kuo, S. C.; Xia, Y.; Bastow, K.; Nakanishi, Y.; Hamel, E.; Lee, K. H. J. Med. Chem. 2000, 43, 4479-4487.

[3]. Birch, H. L.; Buckley, G. M.; Davies, N.; Dyke, H. J.; Frost, E. J.; Gilbert, P. J.; Hannah, D. R.; Haughan, A. F.; Madigan, M. J.; Morgan, T.; Pitt, W. R.; Ratcliffe, A. J.; Ray, N. C.; Richard, M. D.; Sharpe, A.; Taylor, A. J.; Whitworth, J. M.; Williams, S. C. Bioorg. Med. Chem. Lett. 2005, 15, 5335-5339.

[4]. Bonola, G.; Da, R. P.; Magistretti, M. J.; Massarani, E.; Setnikar, I. J. Med. Chem. 1968, 11, 1136-1139.

[5]. Rexall Drug Co., U. S. Patent 3257397, 1966.

[6]. Okumura, K.; Oine, T.; Yamada, Y.; Hayashi, G.; Nakama, M. J. Med. Chem. 1968, 11, 348-351.

[7]. Cohen, E.; Klarberg, B.; Vaughan, J. R. J. Am. Chem. Soc. 1959, 81, 55085509.

[8]. Alagarsamy, V.; Solomon, V. R.; Murugan, M. Bioorg. Med. Chem. 2007, 15, 4009-4015.

[9]. Levin, J. I.; Chan, P. I.; Bailey, T.; Katocs, A. S.; Venkatesan, A. M. Bioorg. Med. Chem. Lett. 1994, 4, 1141-1146.

[10]. Instituto De Angeli S. P. A. French Patent M. 1893, 1963.

[11]. Shulton Inc. U. S. Patent 3265697, 1966.

[12]. Hirose, N.; Kuriyama, S.; Sohda, S.; Sakaguchi, K.; Yamamoto, H. Chem. Pharm. Bull. 1973, 21, 1005-1013.

[13]. Mustazza, C.; Borioni, A.; Sestili, I.; Sbraccia, M.; Rodomonte, A.; Ferretti, R.; Giudice, M. R. D. Chem. Pharm. Bull. 2006, 54, 611-622.

[14]. Hamel, E.; Lin, C. M.; Plowman, J.; Wang, H.; Lee, K.; Paull, K. D. Biochem. Pharmacol. 1996, 51, 53-59.

[15]. Liu, X. W.; Fu, H.; Jiang, Y. Y.; Zhao, Y. F. Angew. Chem., Int. Ed. 2009, 48, 348-351.

[16]. Zhang, Z. H.; Lu, H. Y.; Yang, S. H.; Gao, J. W. J. Comb. Chem. 2010, 12, 643-646.

[17]. Chinigo, G. M.; Paige, M.; Grindrod, S.; Hamel, E.; Dakshanamurthy, S.; Chruszcz, M.; Minor, W.; Milton, L.; Brown, M. L. J. Med. Chem. 2008, 51, 4620-4631.

[18]. Rueping, M.; Antonchick, A. P.; Sugiono, E.; Grenader, K. Angew. Chem., Int. Ed. 2009, 48, 908-910

[19]. Chowdhury, S.; Mohan, R. S.; Scott, J. L. Tetrahedron 2007, 63, 23632389.

[20]. Muzart, J. Adv. Synth. Catal. 2006, 348, 275-295.

[21]. Jain, N.; Kumar, A.; Chauhan, S.; Chauhan, S. M. S. Tetrahedron 2005, 61, 1015-1060.

[22]. Zhao, H.; Malhotra, S. V. Aldrichimica Acta 2002, 35(3), 75-83.

[23]. Darvatkar, N. B.; Bhilare, S. V.; Deorukhkar, A. R.; Raut, D. G.; Salunkhe, M. M. Green Chem. Lett. Rev. 2010, 3, 301-306.

[24]. Greaves, T. L.; Drummond, C. J. Chem. Rev. 2008, 108(1), 206-237.

[25]. Zhao, G.; Jiang, T.; Gao, H.; Han, B.; Juang, J.; Sun, D. Green Chem. 2004, 6, 75-77.

[26]. Raghavendra, G. M.; Ramesha, A. B.; Revanna, C. N.; Nandeesh, K. N.; Mantelingu, K.; Rangappa, K. S. Tetrahedron Lett. 2011, 52, 55715574 .

[27]. Chandrappa, S.; Umashankara, M.; Vinaya, K.; Ananda Kumar, C. S. Rangappa, K. S. Tetrahedron Lett. 2012, 53, 2632-2635.

[28]. Raghavendra, G. M.; Harsha, K. B.; Vinaya, K.; Mantelingu, K.; Rangappa, K. S. Synth. Commun. 2011, 41, 2296-2303.

[29]. Raghavendra, G. M.; Ramesha, A. B.; Revanna, C. N.; Nandeesh, K. N.; Mantelingu, K.; Rangappa, K. S. Tetrahedron Lett. 2011, 52(43), 55715574.

[30]. Revanna, C. N.; Raghavendra, G. M.; JeniferVijay, T. A.; Rangappa, K. S.; Badregowd, D. G.; Mantelingu, K. Chem. Lett. 2014, 43, 178-180.

[31]. Ramesha, A. B.; Raghavendra, G. M.; Nandeesh, K. N.; Rangappa, K. S.; Mantelingu, K. Tetrahedron Lett. 2012, 54, 95-100.

[32]. Jenifer Vijay, T. A.; Nandeesh, K. N.; Raghavendra, G. M.; Rangappa, K. S.; Mantelingu, K. Tetrahedron Lett. 2013, 54, 6533-6537.

[33]. Nandeesh, K. N.; Raghavendra, G. M.; Revanna, C. N.; Jenifer Vijay, T. A.; Rangappa, K. S.; Mantelingu, K. Synth. Commun. 2014, 44, 1103-1110. 